\title{
Malaria outbreaks in China (1990-2013): a systematic review
}

\author{
Guangyu Lu', Shuisen Zhour,3,4, Olaf Horstick' ${ }^{1}$ Xu Wang ${ }^{5}$, Yali Liü ${ }^{6,7}$ and Olaf Müller ${ }^{\text {* }}$
}

\begin{abstract}
Background: China has already achieved remarkable accomplishments in shrinking the malaria burden since the mid-20th Century. The country now plans to eliminate malaria by the year 2020. Looking at the dynamics of malaria outbreaks during the last decades might provide important information regarding the potential challenges of such an elimination strategy and might help to avoid mistakes of the past.

Methods: A systematic review of the published literature (English and Chinese) was conducted to identify malaria outbreaks during the period 1990 until 2013 in China. The main causes of outbreaks as described in these papers were categorized according to whether they were related to population migration, environmental factors, vector and host related factors, and operational problems of the health services.
\end{abstract}

Results: The review identified 36 malaria outbreaks over the 23-year study period, on which sufficient information was available. They mainly occurred in southern and central China involving 12 provinces/autonomous regions. More than half of all outbreaks $(21 / 36,58 \%)$ were attributed at least in part to population migration, with malaria importation to non- or low-endemic areas from high-endemic Chinese areas (13/15) or endemic countries (2/15) having been the most frequent reason $(15 / 21,71 \%)$. Other main causes were problems of the health services $(15 / 36,42 \%)$, in particular poor malaria case management $(10 / 15,67 \%)$, environmental factors $(7 / 36,19 \%)$, and vector and host related factors $(5 / 36,14 \%)$.

Conclusions: Beside a number of other challenges, addressing population movement causing malaria appears to be of particular importance to the national malaria programme. Strengthening of surveillance for malaria and early radical treatment of cases should thus be considered among the most important tools for preventing malaria outbreaks and for the final goal of malaria elimination in China.

Keywords: Malaria, Outbreak, China, Systematic review

\section{Background}

Malaria was highly endemic in China (estimated 30 million annual cases) before the foundation of the People's Republic in 1949 [1]. Tremendous efforts have been put into reducing the malaria burden since the launch of the National Malaria Control Programme in 1955. Despite two major outbreaks in the 1960s and 1970s, respectively, the country showed a steady decrease of its malaria burden [2]. By the end of 1990, there were 117,000 malaria cases reported from China [2]. Over the last two decades, China has further reduced the malaria burden, now with a goal of elimination by 2020 [3,4]. However,

\footnotetext{
*Correspondence: Olaf.Mueller@urz.uni-heidelberg.de

'Institute of Public Health, Medical School, Ruprecht-Karls-University, INF 324, 69120 Heidelberg, Germany

Full list of author information is available at the end of the article
}

sporadic outbreaks have consistently been reported during the last two decades [5-7].

Plasmodium falciparum and Plasmodium vivax are the two main malaria species in China. Falciparum malaria was already restricted to only two provinces (Yunnan, Hainan) by 1998, and vivax malaria accounted for $95 \%$ of the indigenous malaria cases in 2012 [8,9]. Plasmodium vivax is less responsive to control interventions and much more difficult to eliminate than P. falciparum [10]. On the other side, imported falciparum malaria is increasingly seen in many provinces $[8,11]$.

Malaria outbreaks are a clear threat to maintain achievements and to the final goal of elimination in China [12]. They can have various reasons, ranging from environmental causes over parasite/vector characteristics 
to health system factors and human behaviour aspects [13]. Increasing development in China, which is associated with increased mobility, led to malaria outbreaks in areas where local malaria transmission had previously been interrupted. A strengthened surveillance system contributed largely to active investigation and better reporting of these outbreaks. Therefore, a thorough analysis of the specific reasons for these outbreaks will be helpful to improve the effectiveness of the national malaria programme.

A systematic literature review on causes of published malaria outbreaks in China from 1990 until 2013 has thus been conducted.

\section{Methods}

\section{Search strategy}

All published malaria outbreaks within Mainland China between 1 January, 1990 and 12 September, 2013 were systematically retrieved. English articles were searched from Medline, Web of Science, Embase, the Cochrane Central Register of Controlled Trials and Evidence for Policy and Practice Information and coordinating Centre (EPPI-Centre) by using the search terms 'malaria', 'Plasmodium vivax', 'Plasmodium falciparum', 'outbreak', 'resurgence', 're-emergence' 'relapse' and 'China', 'People's Republic of China'. Articles published in Chinese were identified through China National Knowledge Infrastructure (CNKI), China Biology Medicine (CBM), VIP, WanFang database and the website of the Chinese Centre for Disease Control and Prevention by using the same keywords.

\section{Selection criteria Time period}

The study concentrated on the years 1990 until 2013, as data are more complete for this time period and as the reasons for outbreaks from this period are most applicable to the present malaria status in China.

\section{Outbreak causes}

Only studies that discussed potential main causes for malaria outbreaks were included, irrespective of an implementation of active epidemiological investigations or not.

\section{Outbreak definition}

Before 2006, there was no malaria outbreak definition in Chinese guidelines, thus and according to WHO, a malaria outbreak was considered as the occurrence of cases of disease in excess of what would normally be expected in a defined area or season' in the analysis. Since 2006 and according to new Chinese guidelines, malaria outbreaks were more specifically defined as 'an unusual increase or a new occurrence of autochthonous (indigenous or introduced) cases in a certain area' $[14,15]$. In areas of endemic malaria, increase refers to an at least doubling of incidence between two years (starting from at least ten cases per month at village level); in addition at least 3\% of the at-risk population has to contract malaria one or more times in stable endemic areas and at least 1\% in unstable endemic areas. Regarding areas where malaria has already been eliminated (no local transmission for at least three years), an outbreak is defined as at least five indigenous (mosquito-borne malaria from local infections) cases per month or at least one newly introduced (mosquito-borne malaria from an imported infection) falciparum malaria case at village level. In areas where no local malaria transmission has been observed for at least five years, any newly introduced case is defined as an outbreak. The occurrence of only imported cases not having caused secondary cases is not considered an outbreak.

\section{Data extraction and analysis}

All papers found were carefully read and discussed by two authors (GYL, YLL) and those fulfilling the selection criteria were included in the analysis. The characteristics of malaria outbreaks (e g, population, time period, parasite and vector species, malaria epidemiology, and data collection method) were systematically extracted. Outbreaks were listed geographically according to the following four categories from the Action Plan of China Malaria Elimination (2010-2020) [16].

Analytical categories were identified inductively based on the main suggested outbreak causes in every paper. Two authors (GYL, XW) extracted the main causes independently from each paper, and then two authors (GYL, OM) classified them into analytical categories. The content of each paper was systematically checked for all causes relevant to each category. Finally, all causes identified were classified into four main non-overlapping categories: (1) population migration (e g, person migration from low- to high-transmission areas or vice versa); (2) environmental factors (e g, climatic changes, manmade breeding sites and natural disaster); (3) vector and host related factors (e g, increased human-vector contact or vector capacity); and, (4) operational problems of the health services (e g, vector control ceased, problems with case management and weakening of surveillance).

\section{Quality appraisal}

As there is no recognized technical tool for quality appraisal of field reports in non-interventional systematic reviews, a criteria for judgement of the credibility and consistency of included reports was developed. In this analysis, credibility refers to whether the suggested main causes of malaria outbreaks originated only from assertion or speculation without provision of quantitative data from detailed investigation or from evidence-based 
conclusion based on active quantitative or qualitative epidemiological investigation. Consistency of included outbreaks was furthermore crosschecked if outbreaks and their characteristics were reported in more than one publication.

In case of disagreement during paper selection for inclusion, data extraction, analytical category identification and quality appraisal processes, cases were discussed until a consensus had been reached.

\section{Results}

The initial search yielded 1,564 records, of which 1,222 remained after removing duplicates (Figure 1). Among them, 154 articles appeared to describe or allude to malaria outbreaks so the full texts were assessed for eligibility. Of these 154 articles, 59 were excluded because the information in the paper was repeated in more detail in other papers; 29/154 articles describing 17 epidemic events were excluded because they do not meet the outbreak definition. For example, the majority (13/17) of the reported epidemics were based on imported malaria cases with no secondary cases. A few of these epidemics were small family clusters $(2 / 17)$ or caused by parasite transmission through blood donations (2/17). Nine of the 154 articles were excluded because of insufficient information provided; nine were excluded because the reported events occurred before 1990; two articles were excluded because the reported events occurred outside Mainland China. Thus, a total of 46 articles describing 36 outbreaks over the period 19902013 were included in the final analysis (see Additional files 1 and 2).

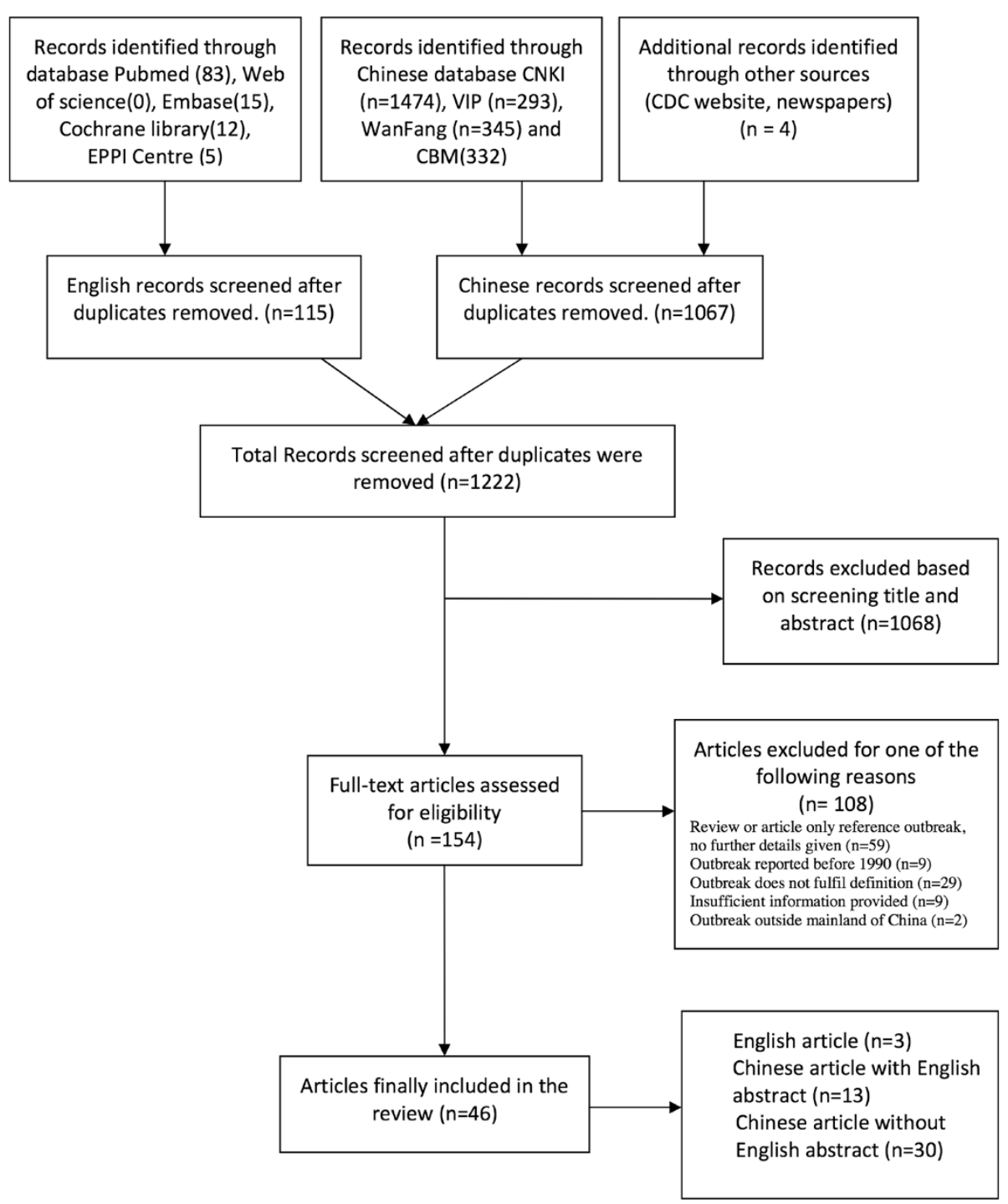

Figure 1 Systematic selection process of malaria outbreak articles in China into the review. 


\section{General outbreak characteristics}

Outbreaks were concentrated in southern China, mainly in Yunnan $(\mathrm{n}=8)$ and Hainan $(\mathrm{n}=10)$ Province, and in the central east (Figure 2). Outbreaks occurred rather homogenous over the study period, but from 2009 onwards, there was a sharp decline with only one further outbreak being reported (Figure 3). This last outbreak was reported from Motuo County located in Tibet Autonomous Region. The malaria species involved into outbreaks were reported in 34/36 articles; $2 / 34$ (6\%) were caused by $P$. falciparum, 20/34 (59\%) by $P$. vivax, and $12 / 34$ (35\%) by both $P$. falciparum and $P$. vivax (Figure 3). Since 2005 there was only one outbreak caused by P. falciparum, which was in Yunnan Province (Additional files 1: Table S1).

\section{Outbreak causes}

The causes of identified outbreaks fell into one of the four general categories, which were not mutually exclusive. Population migration was a main cause in $21 / 36$
(58\%), environmental factors in $7 / 36$ (19\%), changes of vector and host-related factors in 5/36 (14\%), and operational problems of the health services in $15 / 36$ (42\%) of outbreaks (Additional file 2: Table S2). Of 36 reports, 34 were based on active field investigations (2/36 were based on retrospective data analysis). Of these, 19/34 (56\%) implicated population migration, 7/34 (21\%) environmental factors, 5/34 (15\%) changes of vector and host-related factors, and 15/34 (44\%) operational problems of the health services. Seven of 36 (19\%) outbreaks were reported by more than one publication and thus assessed for information consistency. There were no differences in the main outbreak characteristics reported.

\section{Population migration}

There are two subcategories: (1) population movement from an endemic to a non- or low-endemic area $(15 / 21$, $71 \%)$; and, (2) population movement from a non- or low-endemic area to an endemic area $(7 / 21,33 \%)$. The main reasons for outbreaks caused by the first subcategory

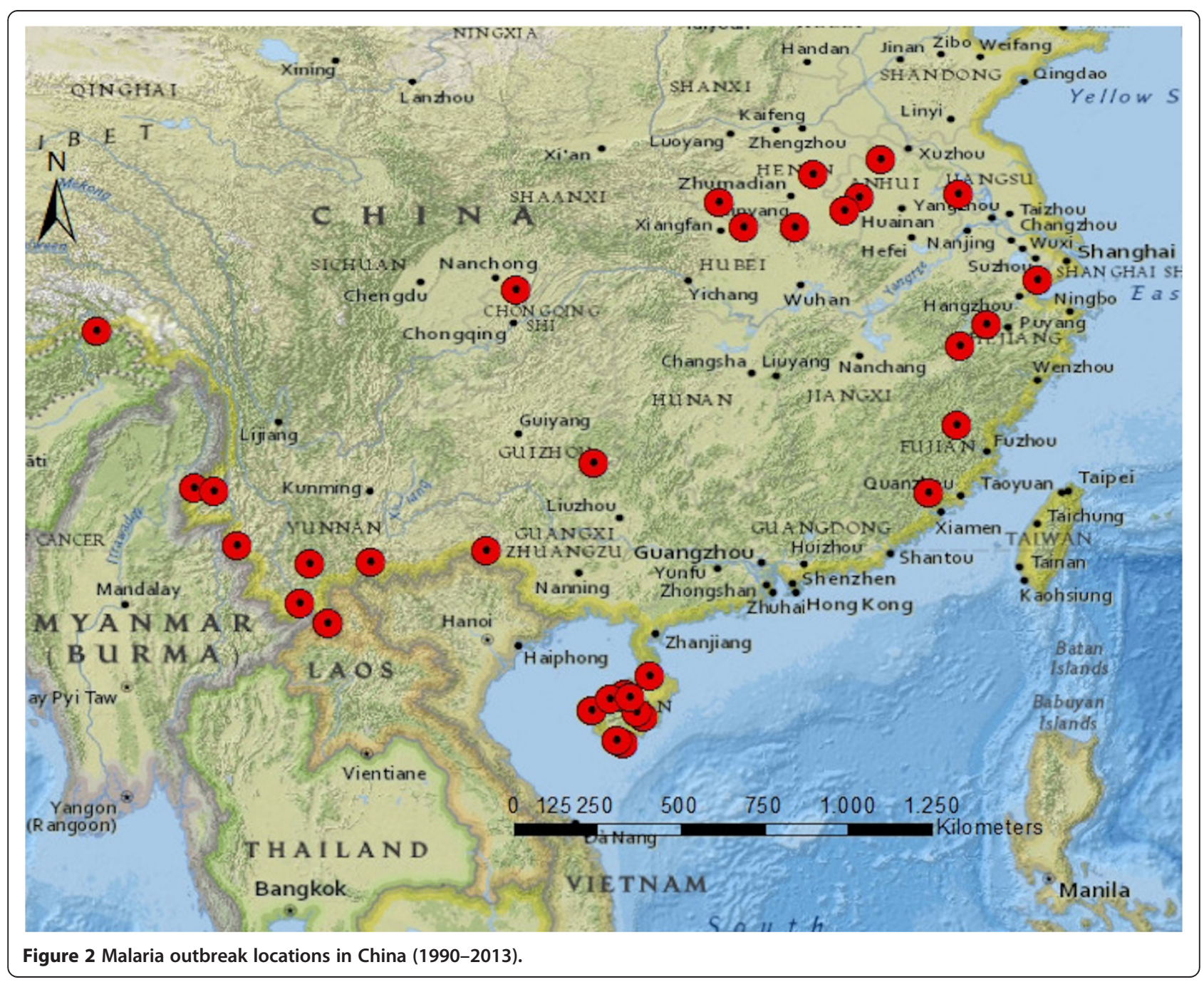




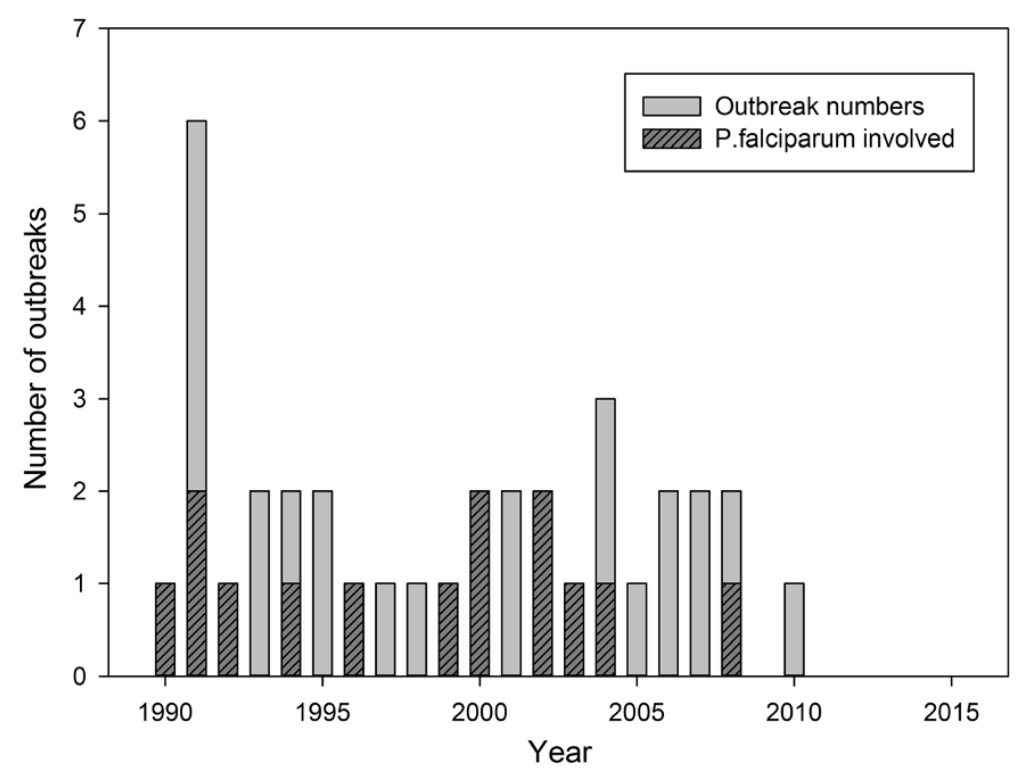

Figure 3 Reported malaria outbreaks in China (1990-2013).

were Chinese workers having migrated to an endemic area (province or country) where they became infected and introduced the malaria parasite to their home on return (7/15), Chinese guest workers from endemic areas (3/15), Chinese tourists returning from an endemic country $(1 / 15)$, and non-specified migration causes (4/15). One example is the return of a local villager from a working trip to Myanmar in 2008, which was followed by a $P$. vivax outbreak in a village in Yunnan Province [17]. The main reason for the second subcategory is population resettlement (2/7) and individual labour movement (5/7). For example in another village of Yunnan Province, an outbreak of 39 malaria cases occurred in 2008, with 31/39 affected migrants from a high-latitude area [18].

\section{Environmental factors}

There are three subcategories: (1) climatic change (1/7, $14 \%)$; (2) man-made breeding sites (3/7, 43\%); and, (3) natural disasters $(3 / 7,43 \%)$. The one outbreak attributed to climate factors was caused by heavy rains. Due to improved breeding opportunities of the main vectors, Anopheles sinensis and Anopheles minius, in a large village in Yunnan Province (28,787 population), which had reported only 27 cases from May to September 1991, 849 malaria cases occurred in October 1991 (596/849 $P$. falciparum) [19]. This was the largest outbreak in Yuanjiang River valley since 1949 [20]. With regard to man-made mosquito breeding sites, the three outbreaks reported were mainly caused by irrigation and deforestation projects. A typical outbreak related to irrigation was documented in a village in Hubei Province, where only 19 malaria cases occurred from 1996 to 2000, but
578 malaria cases were reported in 2001 [21]. The popular conversion of dry areas into wetland for large-scale lotus plantations also contributed to increasing malaria susceptibility. For example, Shangshui County in Henan Province had reported less than five cases annually from 1989-1999, but due to malaria importation in connection with lotus farming, 1,777 malaria cases were reported in 2001 [22,23].

In the year 2005, a large fire disaster in a village in Guizhou Province led to much decreased livestock (e g, cattle) and widespread poverty, which supported increased malaria transmission (as presented also in the discussion). The village had very low annual malaria incidence rates $(3 \sim 4 / 10,000)$ before 2005 , but after the fire, in 2006, there were 46 malaria cases $(611 / 10,000)$ [24]. Other examples of disaster-associated outbreaks are floods which have increased mosquito breeding sites in Anhui province [25].

\section{Vector and host-related factors}

There are two subcategories: (1) increased human-vector contact $(3 / 5,60 \%)$; and (2) increased vector capacity $(2 / 5,40 \%)$. An example of the first subcategory is the reported outbreak in a school of Qiongzhong County, Hainan Province, in 1994. During this event, 72 P. vivax cases occurred in staff members and students because the use of bed nets had been stopped. Before this outbreak, there has been no malaria in this area for three consecutive years [26]. Another example exists from one city located in Henan Province. In this area, there were no malaria cases reported during the period 1992-2002; the first cases were seen again in 2003, and in 2006 a 
large outbreak was reported with 2,889 cases [27]. This outbreak was mainly caused by a combination of livestock reduction and human behaviour change [28]. An example for the second subcategory is an outbreak in a village in Henan Province in 1996, which was in part attributed to the new occurrence of the highly effective vector Anopheles anthropophagus [29].

\section{Operational problems of the health services}

There are three subcategories: (1) ceased vector control measures $(3 / 15,20 \%)$; (2) poor case management $(10 / 15$, $67 \%)$; and, (3) weakening of surveillance $(4 / 15,27 \%)$.

Ceased vector control was attributed to either relaxed vigilance after a large decrease in the malaria burden or to insufficient funding support. One example is a village located in Yunnan Province, where malaria was successfully rolled back due to insecticide spraying. However, only two years after the termination of vector control measures, malaria cases increased again [30]. Problems with case management included insufficient diagnostic and treatment procedures, and stock-out of drugs. The latest outbreak recorded occurred in a small village in Tibet Autonomous Region in 2010 and was caused by stock-out of drugs [31]. Another example is a P. vivax malaria outbreak in a county located in Guizhou Province with 43 cases from June to July 2006, followed by a successive outbreak with 102 cases again from June to July in 2007. While the 2006 outbreak was attributed to a fire disaster (as presented above), the 2007 outbreak was attributed to non-radical treatment of $P$. vivax malaria in the previous year due to a minority population with high prevalence of G6PD deficiency [24].

An example on how weakening of surveillance can play a key role in causing malaria outbreaks was reported from a village in Yunnan Province in the year 2003. The outbreak started at the end of September with a few cases, and the first malaria deaths occurred in October. However, the responsible health authorities did not realize this outbreak until November and 60 cases (63\% P. falciparum) had already occurred [32]. Another example is from Henan Province in 2006, which has been malaria-free from 1992-2002. A general weakening of vigilance of the authorities and the underestimate of the vector capacity of An. sinensis aggravated the malaria situation since the first cases were reported in 2003; there were 2,889 cases reported in 2006 [33].

\section{Discussion}

This is the first systematic review to characterize recent malaria outbreaks in China, based on both Chinese and English publications. The study has analysed the main causes of 36 malaria outbreaks reported since 1990 in China. Malaria outbreaks occurred frequently during this time period in about half of all Chinese provinces, but there was no outbreak in the past five years. This supports the promising development of malaria burden reduction in China in recent years [3,4]. Moreover, a clear shift towards a predominance of $P$. vivax malaria was observed, which supports the assumption of $P$. vivax elimination being more difficult than P. falciparum elimination [34]. Population migration was the most frequently identified outbreak cause, followed by operational problems of the health services, environmental factors and changes of vector and host-related factors.

Population migration clearly is of major importance for malaria outbreaks [13]. The causal pathway can either be that populations move from an endemic to a non- or low-endemic area, leading to new or increased malaria transmission, or from a non- or low-endemic area to an endemic area, increasing the pool of susceptible individuals [35]. The findings from this study show that both pathways play an important role in China. Interestingly and in contrast to the situation in the USA and in European countries, where citizens with migration background often import malaria after having visited friends or relatives in their country of origin [36,37], malaria outbreaks associated with population movements in China were mainly caused by returning export labourers from endemic provinces. These are usually low social class labourers often working under conditions at increased risk of malaria (e g, mining, construction sites, forest work), who are not well protected at night due to poor accommodation, have a low education level and thus lack awareness of malaria risks, have no specific immunity, and often have limited access to health services [38]. Malaria associated with population movements is thus an important threat for elimination, but also for trying to 'hold the line' after having already successfully eliminated malaria $[12,39]$. This needs to be addressed by national programmes, e g, through active screening of returning workers especially in areas at high risk of outbreaks [12]. Early diagnosis and effective treatment would not only avoid unnecessary deaths but also secondary infections.

Operational problems of the health services were identified as another important cause of outbreaks. Here, aspects related to malaria case management were most frequently mentioned, and this concerns mainly $P$. vivax. Chloroquine in combination with primaquine has been used for more than 60 years as radical treatment for vivax malaria and is shown to still be effective in central China [40]. However, compliance and safety aspects with prolonged treatment regimens for $P$. vivax malaria remain a major challenge [41]. Minority populations in China have a high prevalence of G6PD deficiency and are thus at increased risk for haemolysis associated with primaquine treatment [42]. Innovative interventions are thus needed to address this challenge in China if the 
country wants to achieve the goal of malaria elimination by 2020 .

Many formerly endemic areas successfully reduced the malaria burden or even achieved malaria elimination, which was sometimes followed by loosening of vigilance and consequently surveillance by the responsible health services [43]. However, in China surveillance systems have been largely strengthened in recent years and especially after the SARS epidemic [44]. For example, a webbased reporting system of infectious diseases has been implemented since the year 2003 [45]. In order to achieve malaria elimination, a robust surveillance system has been established in China that combines passive and active case detection methods with rapid response measures, including radical treatment and targeted vector control. Moreover and since 2010, China has implemented a '1-3-7' surveillance strategy: one day to report a case, three days to confirm and classify the case, and seven days to conduct a local response and prevent any onward transmission [46]. Since that time, no malaria outbreaks have occurred in China.

Environmental changes such as irrigation or dam projects were also associated with malaria outbreaks in China [47]. However, whether such projects will cause malaria outbreaks depends on various factors. Malaria epidemics are less likely to derive from irrigation projects in high-transmission areas where inhabitants have natural immunity [48]; in contrast, in unstable transmission areas there is high potential for epidemics [49]. In China, most malaria-endemic areas belong to lowtransmission areas. Thus, it is important to strengthen intersectoral cooperation and to establish appropriate health services, including early warning systems together with development projects [50]. Extended rainy seasons and natural disasters such as flooding and earthquakes can also contribute to malaria outbreaks [51]. A particularly interesting outbreak, which is described in this review, was attributed to a major fire disaster, where the reduction of cattle that functioned as a biological barrier largely increased human vector contact [24]. Therefore, outbreak awareness and prevention needs to accompany early responses to such disasters.

The considerable re-emergence of malaria at the beginning of 21st Century, which was mainly observed in central China, was associated with changes in vector capacity of An. sinensis for $P$. vivax [28,52]. In contrast to An. anthropophagus, which has been the main vector in this area in the past, An. sinensis is considered of low epidemiological significance as it usually shows exophilic and non-anthropophilic behaviour [53,54]. Malaria endemicity was sustained in areas where An. sinensis remained the single vector, which has been explained by a significant increase in the transmission capacity of An. sinensis for P. vivax [55,56]. These observations point to the importance of entomological surveys in areas at risk for malaria outbreaks.

This review has some limitations. As these data are from published malaria reports only, it is possible that some outbreaks not documented in the literature have been missed. Another limitation is the fact that clear reasons for outbreaks were frequently not reported in existing publications, and these were consequently not included in the analysis. The findings may also be subject to reporting bias, as report authors may be influenced by their research interests or perhaps by their affiliations (e g, with regional control programmes).

\section{Conclusion}

The findings of this review point to the importance of sustaining quality surveillance for population movements associated with malaria transmission for preventing malaria outbreaks in China. Moreover, further strengthening of environmental and entomological surveillance as well as early case management (e g, radical treatment of vivax cases) will pave the way to achieving the final goal of malaria elimination in China.

\section{Additional files}

Additional file 1: Table S1. Summary of data on malaria outbreaks in China (1990-2013) from systematic review.

Additional file 2: Table S2. Main causes of malaria outbreaks in China (1990-2013).

\section{Competing interests}

The authors declare that they have no competing interests.

\section{Authors' contributions}

The study has been designed by GL and OM. All authors conceived and planned the work that led to the manuscript or played an important role in the acquisition, analysis and interpretation of the data or both. All authors wrote the paper and/or made substantive suggestions for revision. All authors approved the final submitted version.

\section{Acknowledgements}

We acknowledge the financial support of the Deutsche Forschungsgemeinschaft and Ruprecht-Karls-Universität Heidelberg within the funding programme Open Access Publishing, and we also would like to thank Dr Peter Dambach for helpful comments on the Methods as well as for technical assistance with Figure 2.

\section{Author details}

${ }^{1}$ Institute of Public Health, Medical School, Ruprecht-Karls-University, INF 324, 69120 Heidelberg, Germany. ${ }^{2}$ National Institute of Parasitic Diseases, Chinese Centre for Disease Control and Prevention (CDC), Shanghai, China. ${ }^{3}$ Key Laboratory of Parasite and Vector Biology, $\mathrm{MOH}$, Shanghai, China. ${ }^{4} \mathrm{WHO}$ Collaborating Centre for Malaria, Schistosomiasis and Filariasis, Shanghai, China. ${ }^{5}$ Institute of Global Health University College, University College London, London, UK. 'Evidence-Based Medicine Centre, School of Basic Medical Sciences, Lanzhou University, Lanzhou, China. ${ }^{7}$ Key Laboratory of Clinical Translational Research and Evidence-Based Medicine of Gansu Province, Lanzhou, China.

Received: 22 May 2014 Accepted: 8 July 2014

Published: 10 July 2014 


\section{References}

1. Zhou Z: The malaria situation in the People's Republic of China. Bull World Health Organ 1981, 59:931-936.

2. Tang LH, Qian HL, Xu SH: Malaria and its control in the People's Republic of China. Southeast Asian J Trop Med Public Health 1991, 22:467-476.

3. Tang L: Progress in malaria control in China. Chin Med J (Engl) 2000, 113:89-92.

4. Yin JH, Yang MN, Zhou SS, Wang Y, Feng J, Xia ZG: Changing malaria transmission and implications in China towards National Malaria Elimination Programme between 2010 and 2012. PLoS One 2013, 8:e74228.

5. Advisory Committee on Malaria, MOPH: Malaria situation in the People's Republic of China in 1992 (in Chinese). Zhongguo Ji Sheng Chong Xue Yu Ji Sheng Chong Bing Za Zhi 1993, 11:161-164.

6. Advisory Committee on Malaria, MOPH: Malaria situation in the People's Republic of China in 1999 (in Chinese). Zhongguo Ji Sheng Chong Xue Yu Ji Sheng Chong Bing Za Zhi 2000, 18:129-131.

7. Zhou SS, Wang Y, Fang W, Tang LH: Malaria situation in the People's Republic of China in 2007 (in Chinese). Zhongguo Ji Sheng Chong Xue Yu Ji Sheng Chong Bing Za Zhi 2008, 26:401-403.

8. Tang L: Achievements in the research on the prevention and treatment of malaria in China (in Chinese). Zhongguo Ji Sheng Chong Xue Yu Ji Sheng Chong Bing Za Zhi 1999, 17:257-259.

9. Xia ZG, Feng J, Zhou SS: Malaria situation in the People's Republic of China in 2012 (in Chinese). Zhongguo Ji Sheng Chong Xue Yu Ji Sheng Chong Bing Za Zhi 2013, 31:413-418.

10. Greenwood BM: Control to elimination: implications for malaria research. Trends Parasitol 2008, 24:449-454.

11. Lin H, Lu L, Tian L, Zhou S, Wu H, Bi Y, Ho SC, Liu Q: Spatial and temporal distribution of falciparum malaria in China. Malar J 2009, 8:130.

12. Cohen JM, Vallely A, Taleo G, Malefoasi G, Sabot O: Holding the line. In Shrinking the Malaria Map: A Prospectus on Malaria Elimination. Edited by Feachem RGA, Phillips AA, Targett GA. San Francisco: The Global Health Group; 2009:40-60

13. Cotter C, Sturrock HJ, Hsiang MS, Liu J, Phillips AA, Hwang J, Gueye CS, Fullman N, Gosling RD, Feachem RG: The changing epidemiology of malaria elimination: new strategies for new challenges. Lancet 2013, 382:900-911.

14. MOH: Manual of Malaria Prevention and Control (in Chinese). 3rd edition. Bejing: People's Medical Publishing House; 2007.

15. MOH: Sudden Outbreak of Malaria Contingency Plans to Deal With] (in Chinese). China: $\mathrm{MOH} ; 2006$.

16. Action plan of China malaria elimination (2010-2020). http://www.gov. cn/gzdt/att/att/site1/20100526/001e3741a2cc0d67233801.doc

17. Duan YZ, Li SG, Kang XH, Yin SQ, Sun XD: A point-like outbreak caused by secondary transmission from an imported malaria vivax case (in Chinese). Int J Med Parasit Dis 2013, 40:57-59.

18. Pu LQ, Hong $W X$, Zeng $K T$ : Investigation on one endogenous malaria outbreak situation around the border region in the Yunnan Province (in Chinese). J Pathogen Biol 2009, 4:479-480.

19. Dai Q, Guo HP, Ou GR, Yang XB: A report of falciparum malaria outbreak in a Mid-levels district (in Chinese). Dis Surveill 1994, 9:15-16.

20. Bi Y, Chen ZW, Yang YC, Zhu DF: Analysis of a epidemic situation in Yuanjiang river valley of Yunnan province using geographic information system (in Chinese). Chin J Dis Control Prev 2005, 9:197-201.

21. Zuo SL, Chen GY, Huang GQ, Gui AF, Pei SJ, Hu LQ, Chen CY, Yang DL, Zhang WJ: Analysis of the factors causing malaria local outbreak in An. sinensis area, Zaoyang City, Hubei Province (in Chinese). Gonggong Weisheng Yu Yufang Yixue 2002, 13:6-7.

22. Hao JL, Wu WM, Dou CY: Investigation and control of malaria outbreaks in 2001-2003 in Shangshui county (in Chinese). Zhongguo Ji Sheng Chong Xue Yu Ji Sheng Chong Bing Za Zhi 2004, 22:333.

23. Chen JS, Li P, Shang LY LIXH, Zhao JH: Investigation of epidemiological factors in a malaria outbreak in Shangshui County, HeNan Province (in Chinese). HeNan J Prev Med 2004, 15:153-155.

24. Wu NB, Long QQ, Luo T, Wang SH, Zhou J, Tang DL, Deng MM, Li SP: Analysis of the outbreak reasons of malaria for 2 years in Judong Village of Congjiang County in Guizhou] (in Chinese). Modern Prev Med 2008, 35:3629-3631

25. Yang YY: An investigation of local plasmodium vivax malaria outbreak (in Chinese). Anhui J Prev Med 1997, 3:71.
26. Duan JS, Zhang YB, Liang HJ, Chen GY, Zhu MF: Control of a vivax malaria outbreak in Qiongzhong County (in Chinese). Zhongguo Ji Sheng Chong Xue Yu Ji Sheng Chong Bing Za Zhi 1999, 17:270.

27. Zhou GC, Zhang HW, Su YP, Zhou SS: Epidemiologic analysis of malaria outbreak in Yongcheng County of Henan Province in 2006 (in Chinese). J Trop Med 2008, 8:381-383.

28. Pan JY, Zhou SS, Zheng X, Huang F, Wang DQ, Shen YZ, Su YP, Zhou GC, Liu F, Jiang JJ: Vector capacity of Anopheles sinensis in malaria outbreak areas of central China. Parasit Vectors 2012, 5:136.

29. Shang LY, Chen JS, Gu ZC, Zhen X, Liu H, Su YJ AML, Luo MZ, Qian HL, Tang LH: Investigation on malaria outbreak in area of the disease basically eliminated in Henan Province (in Chinese). Chin J Parasit Dis Control 2001, 14:81-83.

30. Che LG, Li XL, Yang YM, Li CF, Zhang YL: A survey on malaria outbreak in Jiabutuo Reclamation Area, YunNan Province (in Chinese). Chin J Parasit Dis Control 1992, 5:81-83.

31. ZhuoMa YJ, Wang HJ, CiRen WM, Wu S, Xu GJ, Zhang R, Hu SL, Xu HM: Investigation of a malaria outbreak in Motuo County, Tibet Autonomous Region (in Chinese). Int J Med Parasit dis 2011, 38:270-272.

32. Yang J, Fang XL, Lin YX, Zou J: Survey and management of a sporadic outbreak of malaria in village on the Myanmar-China border (in Chinese). China Trop Med 2005, 5:477.

33. Zhang HW, Su YP, Zhou GC, Liu Y, Cui J, Wang ZQ: Re-emerging malaria in Yongcheng city of HeNan Province (in Chinese). Chin J Vector Bio Control 2007, 18:42-44.

34. Wells TN, Burrows JN, Baird JK: Targeting the hypnozoite reservoir of plasmodium vivax: the hidden obstacle to malaria elimination. Trends Parasitol 2010, 26:145-151.

35. Prothero RM: Disease and mobility: a neglected factor in epidemiology. Int J Epidemiol 1977, 6:259-267.

36. Jelinek T, TropNetEurop: Imported falciparum malaria in Europe: 2007 data from TropNetEurop. Euro Surveill 2008, 13:13.

37. Cullen KA, Arguin PM: Division of parasitic D, malaria CfGHCfDC, prevention: malaria surveillance-United States, 2011. MMWR Surveill Summ 2013, 62:1-17.

38. Liu Y, Hsiang MS, Zhou H, Wang W, Cao Y, Gosling RD, Cao J, Gao O: Malaria in overseas labourers returning to China: an analysis of imported malaria in Jiangsu Province, 2001-2011. Malar J 2014, 13:29.

39. Zhou G, Sun L, Xia R, Duan Y, Xu J, Yang H, Wang Y, Lee MC, Xiang Z, Yan G, Cui L, Yang Z: Clinical malaria along the China-Myanmar border, Yunnan province, China, January 2011-august 2012. Emerg Infect Dis 2014, 20:681-684

40. Zhu G, Lu F, Cao J, Zhou H, Liu Y, Han ET, Gao Q: Blood stage of Plasmodium vivax in central China is still susceptible to chloroquine plus primaquine combination therapy. Am J Trop Med Hyg 2013, 89:184-187.

41. Galappaththy GN, Tharyan P, Kirubakaran R: Primaquine for preventing relapse in people with Plasmodium vivax malaria treated with chloroquine. Cochrane Database Syst Rev 2013, 10, CD004389.

42. Du CS, Liu LB, Liu B, Tokunaga K, Omoto K: Glucose-6-phosphate dehydrogenase deficiency among three national minorities in Hainan Island, China. Gene Geogr 1988, 2:71-74.

43. Murhandarwati EE, Fuad A, Nugraheni MD, Suyanto S, Wijayanti MA, Widartono BS, Chuang TW: Early malaria resurgence in pre-elimination areas in Kokap Subdistrict, Kulon Progo. Indonesia Malar J 2014, 13:130

44. Kaufman JA: China's heath care system and avian influenza preparedness. J Infect Dis 2008, 197(Suppl 1):S7-S13.

45. Wang L, Wang Y, Jin S, Wu Z, Chin DP, Koplan JP, Wilson ME: Emergence and control of infectious diseases in China. Lancet 2008, 372:1598-1605.

46. Cao J, Sturrock HJW, Cotter C, Zhou SS, Zhou H, Liu YB, Tang LH, Gosling RD, Feachem RGA, Gao Q: Communicating and monitoring surveillance and response activities for malaria elimination: China's "1-3-7" strategy. PLoS Med 2014, 11:e1001642

47. Service MW: Agricultural development and arthropod-borne diseases: a review. Rev Saude Publica 1991, 25:165-178.

48. Boudin C, Robert V, Carnevale P, Ambroise-Thomas P: Epidemiology of Plasmodium falciparum in a rice field and a savanna area in Burkina Faso. Comparative study on the acquired immunoprotection in native populations. Acta Trop 1992, 51:103-111.

49. Keiser J, De Castro MC, Maltese MF, Bos R, Tanner M, Singer BH, Utzinger J: Effect of irrigation and large dams on the burden of malaria on a global and regional scale. Am J Trop Med Hyg 2005, 72:392-406. 
50. Wang DQ, Tang LH, Gu ZC, Zheng X, Jiang WK: Malaria transmission potential in the three gorges reservoir of the Yangtze river, China. Biomed Environ Sci 2013, 26:54-62.

51. Watson JT, Gayer M, Connolly MA: Epidemics after natural disasters. Emerg Infect Dis 2007, 13:1-5.

52. Zhu G, Xia H, Zhou H, Li J, Lu F, Liu Y, Cao J, Gao Q, Sattabongkot J: Susceptibility of Anopheles sinensis to Plasmodium vivax in malarial outbreak areas of central China. Parasit Vectors 2013, 6:176.

53. Sinka ME, Bangs MJ, Manguin S, Chareonviriyaphap T, Patil AP, Temperley WH, Gething PW, Elyazar IR, Kabaria CW, Harbach RE, Hay Sl: The dominant Anopheles vectors of human malaria in the Asia-Pacific region: occurrence data, distribution maps and bionomic precis. Parasit Vectors 2011, 4:89.

54. Liu C: Comparative studies on the role of Anopheles anthropophagus and Anopheles sinensis in malaria transmission in China (in Chinese). Zhonghua liu xing bing xue za zhi 1990, 11:360-363.

55. Xu BL, Su YP, Shang LY, Zhang HW: Malaria control in Henan Province, People's Republic of China. Am J Trop Med Hyg 2006, 74:564-567.

56. Zhou SS, Huang F, Wang JJ, Zhang SS, Su YP, Tang LH: Geographical, meteorological and vectorial factors related to malaria re-emergence in Huang-Huai River of central China. Malar J 2010, 9:337.

doi:10.1186/1475-2875-13-269

Cite this article as: Lu et al:: Malaria outbreaks in China (1990-2013):

a systematic review. Malaria Journal 2014 13:269.

\section{Submit your next manuscript to BioMed Central and take full advantage of:}

- Convenient online submission

- Thorough peer review

- No space constraints or color figure charges

- Immediate publication on acceptance

- Inclusion in PubMed, CAS, Scopus and Google Scholar

- Research which is freely available for redistribution 